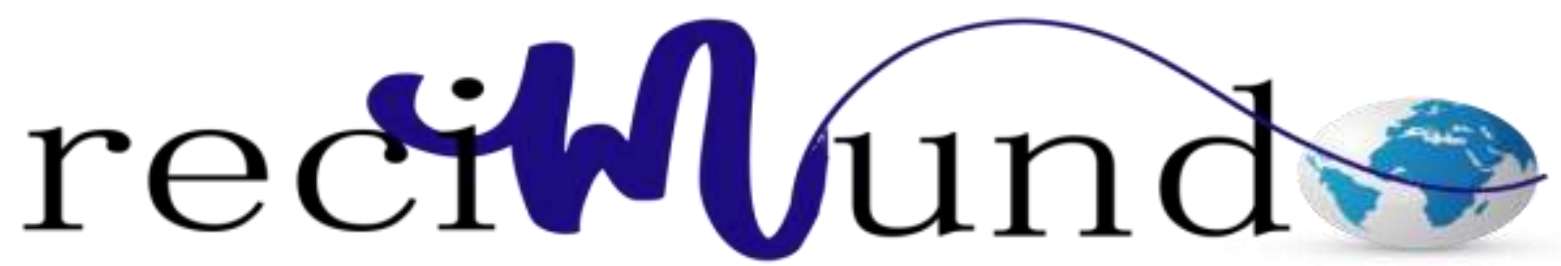

Revista Cientifica Mundo de la Investigación y el Conocimiento

\author{
Ariagna Martínez Pérez a ; Yelennis Galardy Domínguez ${ }^{\text {b; }}$ Sidar Edgardo Solórzano \\ Solórzano ${ }^{\text {c }}$ : Viviana Marianela Quiroz Villafuerte ${ }^{\mathrm{d}}$
}

Cuidado y prevención de parásitos intestinales en infantes

Care and prevention of intestinal parasites in infants

Revista Científica Mundo de la Investigación y el Conocimiento. Vol. 3 núm.3, septiembre, ISSN: 2588-073X, 2019, pp. 444-460

DOI: $10.26820 /$ recimundo/3.(3).septiembre.2019.444-460

URL: http://recimundo.com/index.php/es/article/view/530

Código UNESCO: 3205 Medicina Interna

Tipo de Investigación: Artículo de Revisión

Editorial Saberes del Conocimiento

Recibido: 15/05/2019

Aceptado: 23/06/2019

Publicado: 30/09/2019

Correspondencia: ariagnamartinez@gmail.com

a. Doctora en Medicina; Especialista en primer grado en Medina General; Universidad Estatal del Sur de Manabí; ariagnamartinez@gmail.com

b. Doctora en Medicina; Especialista en primer grado en Medina General; Universidad Estatal del Sur de Manabí; gyelennis@yahoo.com

c. Abogado de los Juzgados y Tribunales de la Republica; Magister en Gerencia Educativa; Universidad Estatal del Sur de Manabí; sidarsolorzano @ hotmail.com

d. Licenciado en Enfermería; Magister en Seguridad clínica del paciente y calidad de atención sanitaria; Universidad Estatal del Sur de Manabí; vivi10_1989@hotmail.com 


\section{Cuidado y prevención de parásitos intestinales en infantes}

Vol. 3, núm. 3., (2019)

Ariagna Martínez Pérez; Yelennis Galardy Domínguez; Sidar Edgardo Solórzano Solórzano;

Viviana Marianela Quiroz Villafuerte

\section{RESUMEN}

Los parásitos intestinales representan una de las mayores preocupaciones en los centros de salud pública de cualquier país, en especial en los países en desarrollo. El siguiente trabajo de investigación, estudia el cuidado y la prevención necesaria de las infecciones causadas por parásitos intestinales presentados en su mayoría en niños, a través, de una revisión literaria que se enfoca al tema de interés y que sirven de apoyo para sustentar el desarrollo del trabajo. El estudio hace mención a los principales parásitos que repercuten en los niños y a los tipos de acuerdo a los órganos que afectan, centrándose en aquellos que inciden principalmente en el aparato digestivo de los infantes. Durante la investigación se observa, que los parásitos intestinales incurren en millones de personas en todo el mundo durante todo el año, por lo que, se debe considerar mantener un adecuado saneamiento ambiental, uso y provisión adecuada del agua ingerida por infantes, así como también, enseñarles buenos hábitos de higiene con el fin de disminuir los riesgos de contraer infecciones intestinales.

Palabras Claves: Infección intestinal; Parásitos; Infección; Niños; Infantes. 


\title{
Cuidado y prevención de parásitos intestinales en infantes
}

Vol. 3, núm. 3., (2019)

Ariagna Martínez Pérez; Yelennis Galardy Domínguez; Sidar Edgardo Solórzano Solórzano; Viviana Marianela Quiroz Villafuerte

\begin{abstract}
Intestinal parasites represent one of the greatest concerns in public health centers in any country, especially in developing countries. The following research work studies the necessary care and prevention of infections caused by intestinal parasites presented mostly in children, through a literary review that focuses on the topic of interest and that serves as support to sustain the development from work. The study mentions the main parasites that affect children and types according to the organs they affect, focusing on those that mainly affect the digestive system of infants. During the investigation it is observed that the intestinal parasites incur millions of people throughout the world throughout the year, so, it should be considered to maintain an adequate environmental sanitation, use and adequate provision of water ingested by infants, as well as, teach them good hygiene habits in order to reduce the risk of getting intestinal infections.
\end{abstract}

Key Words: Intestinal infection; Parasites; Infection; Children; Infants. 


\section{Cuidado y prevención de parásitos intestinales en infantes}

Vol. 3, núm. 3., (2019)

Ariagna Martínez Pérez; Yelennis Galardy Domínguez; Sidar Edgardo Solórzano Solórzano;

Viviana Marianela Quiroz Villafuerte

\section{Introducción.}

Las infecciones parasitarias intestinales se encuentran entre los principales problemas públicos sobre todo en los países pobres, es una de las preocupaciones socioeconómicas y de salud más frecuentes que afecta a los institutos de salud y de los cuales se debe tener gran precaución y mayor atención por parte de los entes de estado.

Existen diferentes tipos de parásitos que se alojan en el área intestinal, entre los que se encuentran: Ascaris Lumbricoides, Anquilostomas, Trichuris Trichiura, Esquistosomiasis, Entamoeba Histolytica y Giardia lamblia (OMS, 2002). En el mundo, millones de personas se ven afectadas por parásitos, sin embargo, los niños son los más vulnerables a contraer infecciones intestinales, debido a la exposición al suelo, contacto con otros niños en escuelas o cuidados, entre otros.

El autor (Rao V, 2006) en su estudio expresa que, en los niños, las infecciones parasitarias intestinales, en particular la helmintiasis transmitida por el suelo, es la causa común de los problemas de salud en los países tropicales. Los niños están más predispuestos a infecciones intestinales graves que las personas adultas, ya que, sus sistemas inmunológicos aún no están completamente desarrollados.

La infección con helmintos está asociada a una considerable tasa de mortalidad y morbilidad en niños, adicionalmente, se ha comprobado que este tipo de infección afecta la salud mental de los niños, su desarrollo, su crecimiento y forma física y a su vez, predispone a los infantes a otros agentes infecciosos. 


\section{Cuidado y prevención de parásitos intestinales en infantes}

Vol. 3, núm. 3., (2019)

Ariagna Martínez Pérez; Yelennis Galardy Domínguez; Sidar Edgardo Solórzano Solórzano; Viviana Marianela Quiroz Villafuerte

Varios factores como condiciones climáticas, saneamiento deficiente, agua potable insegura, y la falta de servicios higiénicos son los principales contribuyentes a la alta prevalencia de parásitos intestinales en los países tropicales y subtropicales. Además, la falta de conciencia sobre el modo de transmisión de las infecciones parasitarias aumenta el riesgo de infección. (Mahfouz AAR, 2016)

Por lo tanto, se debe tener una mejor comprensión de los factores anteriores, así como también, tomar conciencia social, cultural, comportamental y comunitaria para disminuir los riesgos que afectan la epidemiologia y control de los parásitos intestinales diseñando un plan efectivo que ayude a crear estrategias frente al manejo de estas enfermedades.

Son pocos los estudios que han demostrado la magnitud de infecciones parasitarias intestinales existentes en América Latina en niños de edad escolar y en menores de cinco años, la información acerca del conocimiento básico de transmisión y prevención de infecciones intestinales es limitada.

Por tal motivo, este trabajo investigativo se enfoca en evaluar la prevalencia de las infecciones parasitarias intestinales en infantes, las causas, los efectos, modos de transmisión, prevención y los tratamientos requeridos, con la finalidad de servir de apoyo y brindar información importante para futuras investigaciones.

\section{Metodología.}

Para el desarrollo de este proceso investigativo, se plantea como metodología la encaminada hacia una orientación científica particular que se encuentra determinada por la 


\section{Cuidado y prevención de parásitos intestinales en infantes}

Vol. 3, núm. 3., (2019)

Ariagna Martínez Pérez; Yelennis Galardy Domínguez; Sidar Edgardo Solórzano Solórzano;

Viviana Marianela Quiroz Villafuerte

necesidad de indagar en forma precisa y coherente una situación, en tal sentido (Davila, 2015) define la metodología "como aquellos pasos previos que son seleccionados por el investigador para lograr resultados favorables que le ayuden a plantear nuevas ideas”. (p.66)

Lo citado por el autor, lleva a entender que el desarrollo de la acción investigativa busca simplemente coordinar acciones enmarcadas en una revisión bibliográfica con el fin de complementar ideas previas relacionadas "Cuidado y prevención de parásitos intestinales en infantes", a través, de una revisión de literatura, para así finalmente elaborar un cuerpo de consideraciones generales que ayuden a ampliar el interés propuesto.

\section{Tipo de Investigación.}

Dentro de toda práctica investigativa, se precisan acciones de carácter metodológico mediante las cuales, se logra conocer y proyectar los eventos posibles que la determinan, así como las características que hacen del acto científico un proceso interactivo ajustado a una realidad posible de ser interpretada. En este sentido, se puede decir, que la presente investigación corresponde al tipo documental, definido por Castro (2016), "se ocupa del estudio de problemas planteados a nivel teórico, la información requerida para abordarlos se encuentra básicamente en materiales impresos, audiovisuales y /o electrónicos”. (p.41).

En consideración a esta definición, la orientación metodológica permitió la oportunidad de cumplir con una serie de actividades inherentes a la revisión y lectura de diversos documentos donde se encontraron ideas explicitas relacionadas con los tópicos encargados de identificar a cada característica insertada en el estudio. Por lo tanto, se realizaron continuas interpretaciones con el 


\section{Cuidado y prevención de parásitos intestinales en infantes}

Vol. 3, núm. 3., (2019)

Ariagna Martínez Pérez; Yelennis Galardy Domínguez; Sidar Edgardo Solórzano Solórzano; Viviana Marianela Quiroz Villafuerte

claro propósito de revisar aquellas apreciaciones o investigaciones propuestas por diferentes investigadores relacionadas con el tema de interés, para luego dar la respectiva argumentación a los planteamientos, en función a las necesidades encontradas en la indagación.

\section{Fuentes Documentales.}

El análisis correspondiente a las características que predomina en el tema seleccionado, llevan a incluir diferentes fuentes documentales encargadas de darle el respectivo apoyo y en ese sentido cumplir con la valoración de los hechos a fin de generar nuevos criterios que sirven de referencia a otros procesos investigativos. Para (CASTRO, 2016) las fuentes documentales incorporadas en la investigación documental o bibliográfica, "representa la suma de materiales sistemáticos que son revisados en forma rigurosa y profunda para llegar a un análisis del fenómeno".(p.41). Por lo tanto, se procedió a cumplir con la realización de una lectura previa determinada para encontrar aquellos aspectos estrechamente vinculados con el tema, con el fin de explicar mediante un desarrollo las respectivas apreciaciones generales de importancia.

\section{Técnicas para la Recolección de la Información.}

La conducción de la investigación para ser realizada en función a las particularidades que determinan a los estudios documentales, tiene como fin el desarrollo de un conjunto de acciones encargadas de llevar a la selección de técnicas estrechamente vinculadas con las características del estudio. En tal sentido, (Bolívar, 2015), refiere, que es "una técnica particular para aportar ayuda a los procedimientos de selección de las ideas primarias y secundarias”. (p. 71). 


\section{Cuidado y prevención de parásitos intestinales en infantes}

Vol. 3, núm. 3., (2019)

Ariagna Martínez Pérez; Yelennis Galardy Domínguez; Sidar Edgardo Solórzano Solórzano;

Viviana Marianela Quiroz Villafuerte

Por ello, se procedió a la utilización del subrayado, resúmenes, fichaje, como parte básica para la revisión y selección de los documentos que presentan el contenido teórico. Es decir, que mediante la aplicación de estas técnicas se pudo llegar a recoger informaciones en cuanto a la revisión bibliográfica de los diversos elementos encargados de orientar el proceso de investigación. Tal como lo expresa, (Bolívar, 2015) "las técnicas documentales proporcionan las herramientas esenciales y determinantes para responder a los objetivos formulados y llegar a resultados efectivos" (p. 58). Es decir, para responder con eficiencia a las necesidades investigativas, se introdujeron como técnica de recolección el método inductivo, que hizo posible llevar a cabo una valoración de los hechos de forma particular para llegar a la explicación desde una visión general.

Asimismo, se emplearon las técnicas de análisis de información para la realización de la investigación que fue ejecutada bajo la dinámica de aplicar diversos elementos encargados de determinar el camino a recorrer por el estudio, según, (Bolívar, 2015) las técnicas de procesamiento de datos en los estudios documentales "son las encargadas de ofrecer al investigador la visión o pasos que debe cumplir durante su ejercicio, cada una de ellas debe estar en correspondencia con el nivel a emplear" (p. 123). Esto indica, que para llevar a cabo el procesamiento de los datos obtenidos una vez aplicado las técnicas seleccionadas, tales como: fichas de resumen, textual, registros descriptivos entre otros, los mismos se deben ajustar al nivel que ha sido seleccionado

\section{Resultados.}

Es realmente alarmante lo complicadas que pueden llegar a ser las infecciones intestinales si no se les presta la atención y el cuidado debido y a tiempo, la cantidad de riesgo a los cuales son 


\section{Cuidado y prevención de parásitos intestinales en infantes}

Vol. 3, núm. 3., (2019)

Ariagna Martínez Pérez; Yelennis Galardy Domínguez; Sidar Edgardo Solórzano Solórzano; Viviana Marianela Quiroz Villafuerte

expuestos los niños los hace más vulnerables a contraer esta clase de infecciones, sin embargo, llevar a cabo el cuidado y la prevención adecuada es la clave para evitar o disminuir estos riesgos.

En un estudio realizado por la OMS (Organización Mundial de la Salud) en el año 2012, se estimó que los parásitos Ascaris lumbricoides, Anquilostomas y Trichuris trichiura pertenecientes al tipo de parasitos Helmintiasis infectan 1,450 millones, 1,300 millones y 1,050 millones de personas en todo el mundo, respectivamente, mientras que la Esquistosomiasis, también familia de la helmintiasis infecta a más de 200 millones de personas, el parasito Entamoeba histolytica y Giardia lamblia se estima que infecta a 60 millones y 200 millones de personas.

Las parasitosis intestinales son infecciones intestinales que pueden producirse por la ingestión de quistes de protozoos, huevos o larvas de gusanos o por la penetración de larvas por vía transcutánea desde el suelo. Cada uno de ellos va a realizar un recorrido específico en el huésped y afectará a uno o varios órganos, por lo que se pueden clasificar según el tipo de parásito y la afectación que provoquen en los distintos órganos y sistemas. (Botero D, 2013).

Los protozoos son organismos unicelulares microscópicos que pueden ser de vida libre o de naturaleza parasitaria, capaces de multiplicarse en los seres humanos, lo cual contribuye a su supervivencia y también permite que se desarrollen infecciones graves a partir de tan solo un organismo. La transmisión de protozoos que viven en el intestino humano a otro ser humano generalmente ocurre por la vía fecal-oral (por ejemplo, alimentos o agua contaminados o contacto de persona a persona). Los protozoos que viven en la sangre o tejidos humanos se transmiten a otros seres humanos mediante un artrópodo vector (por ejemplo, por la picadura de un mosquito o 


\section{Cuidado y prevención de parásitos intestinales en infantes}

Vol. 3, núm. 3., (2019)

Ariagna Martínez Pérez; Yelennis Galardy Domínguez; Sidar Edgardo Solórzano Solórzano;

Viviana Marianela Quiroz Villafuerte

jején. Los helmintos son organismos grandes multicelulares que por lo general se observan a simple vista cuando son adultos. Al igual que los protozoos, los helmintos pueden ser de vida libre o de naturaleza parasitaria. En su forma adulta, los helmintos no pueden multiplicarse en los seres humanos. (CDC, 2016)

En la tabla 1 se presenta la clasificación de las principales parasitosis intestinales (potozoos y helmintos) que generalmente afectan a los niños y que repercuten en el aparato digestivo.

Tabla N¹. Clasificación de las principales parasitosis intestinales.

\section{Protozoos}

1. Afectación exclusivamente digestiva: Giardiasis: Giandia lamblia

2. Afectación digestiva y potencialmente en tejidos:

a. Amebiasis: Entamoeba histolytica/dispar

b. Criptosporidiasis: Cryptosporiditum

Helmintos

1. Nemathelmintos o nematodos o gusanos cilíndricos:

a. Afectación exclusivamente digestiva:

- Oxiuriasis: Enterobius vermicularis

- Tricocefalosis: Trichuris trichura

b. Afectación digestiva y pulmonar:

- Ascariosis: Ascaris lumbricoides

- Anquilostomiasis o uncinariasis:

- Ancylostoma duodenale

- Necator americanus

c. Afectación cutánea, digestiva y pulmonar:

- Estrongiloidiasis: Strongyloides stercoralis

2. Plathelmintos o cestodos o gusanos planos

a. Afectación exclusivamente digestiva:

- Himenolepiasis: Hymenolepis nana

- Teniasis: Taenia saginata y solium

b. Posibilidad de afectación digestiva y potencialmente en tejidos:

- Teniasis: Taenia solium: Cisticercosis

Fuente: (Botero D, 2013). 


\section{Cuidado y prevención de parásitos intestinales en infantes}

Vol. 3, núm. 3., (2019)

Ariagna Martínez Pérez; Yelennis Galardy Domínguez; Sidar Edgardo Solórzano Solórzano; Viviana Marianela Quiroz Villafuerte

En la tabla se observa que muchos de estos parásitos además de afectar el aparato digestivo de los niños también afectan otros órganos como los pulmones y tejidos, es por este motivo que se considera sumamente importante tratar cualquier infección intestinal a tiempo una vez se detecta la presencia de algún parasito en el cuerpo.

En un estudio efectuado por el autor (Shakya B, 2012) donde se realizaron exámenes de heces a una población de 165 niños en edades comprendidas desde los 3 hasta los 14 años en un colegio ubicado en Nepal, ademas de estudios socioeconómicos para cada uno de los niños, se observó que, de las 165 muestras de heces, 23 (13.9\%) muestras tuvieron una o más parásitos intestinales.

Los niños tuvieron una tasa positiva más baja (10.3\%) que las niñas (19.1\%) (P>0.05), el parasito Entamoeba histolytica $(36,0 \%)$ fue el parásito más frecuente seguido de Ascaris lumbricoides (28.0\%). El otro de los parásitos detectados fueron Hymenolepis nana, anquilostoma y Giardia lamblia.

\section{Clínica y Diagnostico.}

Los parásitos pueden estar en el huésped sin presentar alguna sintomatología portadora, simplemente se observan síntomas de cuadro clínico típico y característico. Los síntomas se presentan de acuerdo al número, tamaño, actividad y toxicidad del parasito de su situación en el huésped y de la capacidad inmunológica de la persona y del ciclo del parasito.

No existe correlación entre la presencia de síntomas gastrointestinales y la presencia de parasitosis. Con frecuencia, cursan con sintomatología variada: dispepsia, diarrea, estreñimiento, 


\section{Cuidado y prevención de parásitos intestinales en infantes}

Vol. 3, núm. 3., (2019)

Ariagna Martínez Pérez; Yelennis Galardy Domínguez; Sidar Edgardo Solórzano Solórzano;

Viviana Marianela Quiroz Villafuerte

dolor abdominal difuso, etc. y, en muchas ocasiones, son asintomáticas o poco sintomáticas. La anemia y la eosinofilia pueden alertar de la presencia de helmintiasis. (Echeverría, 2012)

El diagnóstico suele realizarse con la detección de parásitos, larvas o huevos en las heces. La cantidad de parásitos (en cualquiera de sus formas: protozoos, huevos o larvas) que se elimina por heces varía enormemente en un mismo individuo, incluso de un día para otro, por lo que se recomienda la recogida de tres muestras habitualmente en días alternos, si bien determinados parásitos con cargas bajas pueden requerir un número superior (Martín del Barco OH, 2009). Se recomienda en cualquier caso, si el paciente continúa con síntomas y persiste la sospecha clínica, recoger tantas muestras como fueran necesarias. Puede ser útil instruir al paciente para que recoja los elementos parasitarios detectados en un bote hermético con suero fisiológico (por ejemplo, anillos de tenia).

No se debe suministrar tratamiento con antibióticos especialmente tetraciclinas y metronidazol antes de la recogida de las muestras así como el bismuto, las papillas de bario y el aceite mineral, ya que pueden interferir igualmente. Para facilitar la visualización microscópica, se recomienda seguir durante los días previos una dieta exenta de grasas y fibras. (Fisterra, 2006)

El cribaje sistemático de parásitos en heces en inmigrantes de zonas con alta prevalencia, es un tema controvertido, entre otras razones, porque los parásitos suelen desaparecer espontáneamente con el tiempo, no afectan a la población local y la rentabilidad de una sola muestra de heces es baja. Parece razonable hacerlo en aquellos inmigrantes recientes (dos - cinco años de estancia) y si existen anemia o eosinofilia. (Gasteiz, 2008) 


\section{Cuidado y prevención de parásitos intestinales en infantes}

Vol. 3, núm. 3., (2019)

Ariagna Martínez Pérez; Yelennis Galardy Domínguez; Sidar Edgardo Solórzano Solórzano; Viviana Marianela Quiroz Villafuerte

Tratamientos, cuidados y prevención.

Para prevenir las infecciones parasitarias se tiene como objetivo principal mejora las capacidades de salud, nutrición y aprendizaje infantil, así como también, para mejorar la vida de las mujeres y su capacidad de cuidado, eficiencia laboral y económica productividad.

La evidencia reciente confirma que una significativa reducción de la carga de morbilidad debida a estos agentes infecciosos se puede lograr regulando el tratamiento antihelmíntico dirigido a todos los grupos de alto riesgo. Actualmente, una serie de medicamentos son incluidos en la lista modelo de la OMS, estos medicamentos se ofrecen como tratamiento de dosis única de soiltransmitido, helmintos y esquistosomiasis. (OMS, 2011)

Se ha estimado que la mayor parte de la carga de la enfermedad debido a Helmintos y esquistosomiasis transmitidos por el suelo se puede prevenir en comunidades de alta prevalencia tratando a tiempo a los niños en edad preescolar y escolar. La (OMS, 2011) expresa que tanto albendazol (400 mg) como mebendazol (500 mg) ofrecen la ventaja adicional de que puede ser administrado como una sola tableta para todas las personas mayores de 2 años. Estas drogas son efectivas, bien toleradas y económicas y además no necesita presentar síntomas de infección para ser administradas a los pacientes.

Puede administrarse de manera segura después del primer trimestre del embarazo. También son seguros para niños entre 1 y 2 años de edad (aunque la dosis del albendazol debe reducirse a 200 mg). Los estudios en mujeres embarazadas han demostrado que la desparasitación es beneficiosa durante el curso del embarazo. 


\section{Cuidado y prevención de parásitos intestinales en infantes}

Vol. 3, núm. 3., (2019)

Ariagna Martínez Pérez; Yelennis Galardy Domínguez; Sidar Edgardo Solórzano Solórzano;

Viviana Marianela Quiroz Villafuerte

El tratamiento antihelmíntico se puede dispensar, a través, de servicios de salud materna infantil, clínicas prenatales y salud escolar. El control común y permanente se puede lograr, a través, de un tratamiento regular acompañado de intervenciones preventivas claves a largo plazo para romper las rutas de transmisión tomando en consideración los siguientes aspectos:

- Provisión y uso de un seguro y adecuado suministro de agua.

- Mejora del saneamiento ambiental.

- Buenos hábitos de saneamiento e higiene.

Sobre la base de las últimas pruebas y de un análisis de riesgo-beneficio que tuvo en cuenta la seguridad de los medicamentos y el daño potencial causado por estas infecciones parasitarias si no se tratan, por lo que, se recomienda la consulta informal de la OMS para promover jornadas de desparasitación incluyendo niños de 12 meses en adelante, mujeres embarazadas y adolescentes.

\section{Conclusiones.}

De acuerdo a los estudios presentados en el desarrollo de la investigación se considera, que las infecciones causadas por parásitos son una de las razones más comunes y de las más recurrentes en los centros de salud pública tomando en consideración que la población de niños es la más vulnerable a contraer esta clase de infecciones.

Es realmente alarmante lo complicadas que pueden llegar a ser este tipo de infecciones si no se les presta la atención y los cuidados necesarios para prevenir y disminuir los riesgos que los parásitos pueden desencadenar. Durante la investigación se observó que los parásitos afectan a una 


\section{Cuidado y prevención de parásitos intestinales en infantes}

Vol. 3, núm. 3., (2019)

Ariagna Martínez Pérez; Yelennis Galardy Domínguez; Sidar Edgardo Solórzano Solórzano; Viviana Marianela Quiroz Villafuerte

gran cantidad de habitantes en el mundo en todo el año, tomando en cuenta que los tipos que más repercuten en la aparición de los huéspedes infantes, son los potozoos y los helmintos que, a pesar de afectar mayormente el área intestinal, algunos de ellos, en ocasiones, afectan los órganos pulmonares y tejidos.

Las escuelas suelen ser sitios de mayor exposición a parásitos para los niños, de acuerdo al estudio presentado en los resultados, se detectó que el parasito Entamoeba histolytica fue el más frecuente, los exámenes de heces arrojaron que las niñas eran quienes más tenían dicho parasito. Debido a esto, se recomienda mantener un mayor saneamiento ambiental, uso seguro y provisión del agua que ingieren los niños en las escuelas y buenos hábitos de higiene a modo de disminuir los riesgos de infecciones parasitarias.

Las infecciones parasitarias suelen ser asintomáticas, es recomendable que si se presenta un cuadro clínico típico realizar exámenes de heces para detectar la presencia temprana de algún parasito y poder realizar el tratamiento necesario.

\section{Bibliografía.}

Bolívar, J. (2015). Investigación Documental. México. Pax.

Botero D, R. M. (2013). Parasitosis humanas. Medellin: Corporación para Investigaciones Biológicas (CIB) $4^{\mathrm{a}}$ ed.

Castro, J. (2016). Técnicas Documentales. México. Limusa. 


\section{Cuidado y prevención de parásitos intestinales en infantes}

Vol. 3, núm. 3., (2019)

Ariagna Martínez Pérez; Yelennis Galardy Domínguez; Sidar Edgardo Solórzano Solórzano;

Viviana Marianela Quiroz Villafuerte

CDC. (27 de Octubre de 2016). Centro para el control y la prevencion de Enfermedades.

Recuperado el 7 de Junio de 2019, de https://www.cdc.gov/parasites/es/about.html

Davila, A. (2015). Diccionario de Términos Científicos. . Caracas: Editorial Oasis.

Echeverría, J. (2012). PARASITOSIS INTESTINALES. INFAC, 6-15.

Fisterra. (26 de Agosto de 2006). Parasitosis. Guías Clínicas Fisterra. . Recuperado el / de Junio de 2019, de http://www.fisterra.com/fisterrae/ (Accedido el 1/12/2008).

Gasteiz, V. (8 de Noviembre de 2008). Recomendaciones para la Asistencia Médica al Adulto Inmi- grante. Plan Vasco de Inmigración. Osakidetza. Servicio Central de Publicaciones del Gobierno Vasco. Recuperado el 7 de Junio de 2019, de http://www.ejgv.euskadi.net/r53-2291/es/ contenidos/informacion/publicaciones_osk/es_6574/adjuntos/asis- tenciaInmigrante_c.pdf

Mahfouz AAR, E.-M. H. (2016). Determinantes ecológicos de parásitos intestinales. Infecciones entre niños en edad preescolar. Diario de Tropical, 44-89.

Martín del Barco OH, Á. P. (2009). Parasitosis intestinal. FMC.

OMS. (2011). Guía para gestores de programas de control. Control de helmintos en niños en edad escolar. Ginebra.

OMS, C. d. (2002). Prevención y control de Esquistosomiasis y helmintiasis transmitidas por el suelo. Serie de Informes Técnicos de la OMS, OMS, Buenos Aires. 


\section{Cuidado y prevención de parásitos intestinales en infantes}

Vol. 3, núm. 3., (2019)

Ariagna Martínez Pérez; Yelennis Galardy Domínguez; Sidar Edgardo Solórzano Solórzano; Viviana Marianela Quiroz Villafuerte

Rao V, S. A. (2006). Malnutrition and high childhood mortality among the Onge tribe of the Andaman and Nicobar Islands. (págs. 19-25). Mexico: Public Health Nutrition.

Salud, O. M. (2012). Comité de Expertos de la OMS. Prevención y control de Esquistosomiasis y helmintiasis transmitidas por el suelo. Santiago de Chile: Serie de Informes Técnicos de la OMS.

Shakya B, S. S. (2012). Intestinal parasitic infection among school children. Journal of Nepal Health Research Council , 3-5. 